\title{
Dynamic behaviour of planar rigid multi-body systems including revolute joints with clearance
}

\author{
P Flores $^{1 *}$, J Ambrósio ${ }^{2}$, J C P Claro ${ }^{1}$, and H M Lankarani ${ }^{3}$ \\ ${ }^{1}$ Departamento de Engenharia Mecânica, Universidade do Minho, Guimarães, Portugal \\ ${ }^{2}$ Instituto de Engenharia Mecânica (IDMEC), Instituto Superior Técnico, Lisboa, Portugal \\ ${ }^{3}$ Mechanical Engineering Department, Wichita State University, Kansas, USA
}

The manuscript was received on 25 October 2006 and was accepted after revision for publication on 21 February 2007.

DOI: 10.1243/14644193JMBD96

\begin{abstract}
This paper deals with a general methodology to assess the influence of the clearance size and the friction coefficient on the dynamic response of planar rigid multi-body systems including revolute joints with clearance. When there is a clearance in a revolute joint, impacts between the journal and the bearing can occur, and consequently, local deformations take place. The impact is internal and the response of the system is performed using a continuous contact force model. The friction effect because of the contact between joint elements is also included. The dynamic response of the systems is obtained numerically by solving the constraint equations and the contact-impact forces produced in the clearance joint, simultaneously with the differential equations of motion and a set of initial conditions. Numerical results for two simple mechanisms with revolute clearance joints are presented and discussed. In the present work, the clearance size and friction effects are analysed separately. Through the use of Poincaré maps, both periodic and chaotic responses of the systems are observed. The results predict the existence of the periodic or regular motion at certain clearance sizes and friction coefficients and chaotic or non-linear in other cases.
\end{abstract}

Keywords: multi-body systems, clearance joints, friction coefficient, dynamic behaviour

\section{INTRODUCTION}

Over the last two decades, the dynamic modelling of multi-body systems in general has been recognized as an important tool in the analysis, design, optimization, control, and simulation of complex mechanisms. Clearance, friction, impact and other phenomena associated with real joints are routinely ignored. Therefore, the dynamics of mechanical systems is often conducted under the assumption that the joints are ideal or perfect. The increasing requirement for high-speed machines and mechanisms precision, it becomes imperative to treat the joint in a realistic way, that is, considered the joint clearances. Joint clearances can affect the dynamic performance of the mechanisms significantly.

\footnotetext{
* Corresponding author: Departmento de Engenharia Mecânica, Universidade do Minho, Campus deAzurém, Guimaraes 4800-058, Portugal.email:pflores@dem.uminho.pt
}

In general, the dynamic analysis of mechanical systems is assumed that the kinematic joints are ideal or perfect, that is, clearance, local deformations, wear, and lubrication effects are neglected. However, in a real mechanical joint a clearance is always present. Such clearance is necessary to allow the relative motion between the connected bodies as well as to permit the components assemblage. For instance, in a journal-bearing joint there is a radial clearance allowing for the relative motion between the journal and the bearing. The presence of such joint clearance leads to degradation of the performance of mechanical systems in virtue of the impact forces that take place. On the other hand, no matter how small the clearance is, it can lead to vibration and fatigue phenomena, premature failure and lack of precision or even random overall behaviour. If there is no lubricant in the joint, impacts occur in the system and the corresponding impulses are transmitted throughout the mechanical system.

The degradation of the performance of mechanical systems with joint clearances has been recognized 
for a number of years [1-4]. It is well known that the forces within joints with realistic amounts of clearance, because of clearance impacts, are much higher than would be calculated if the joint clearances were neglected [5]. High joint impact forces can also cause early failure; however, totally eliminating connections clearance from a mechanical system to minimize joint impacts is usually either expensive or impossible.

The main purpose of the present work is to study the dynamic behaviour of planar rigid multi-body systems with revolute clearance joints. In a revolute clearance joint, if there is no lubricant, the journal moves freely inside the bearing boundaries until it reaches the bearing wall. When the journal and the bearing are in contact, deformation takes place at the contact zone resulting in a contact force normal to the plane of collision. The force is proportional to the local indentation and relative penetration velocity, and is modelled by a non-linear continuous force model proposed by Lankarani and Nikravesh [6]. The friction effects due to the relative tangential velocity on the contact zone are also modelled accordingly to modified Coulomb friction law [7]. These normal and tangential forces are introduced into the equations of motion of the system during the period of contact. This approach provides accurate results, in so far the equations of motion are integrated over the period of contact. It thus accounts for the changes in the configuration and the velocities of the system during that contact.

Two elementary planar mechanisms with revolute joint clearances are used as numerical examples to illustrate the application of the presented methodology to the dynamic analysis of rigid multi-body systems.

\section{STATE OF THE ART}

The subject of the representation of imperfect joints has attracted the attention of a large number of researchers that produced a number of theoretical and experimental works devoted to the dynamic simulation of mechanical systems with joint clearances $[\mathbf{2}-\mathbf{5}, \mathbf{8}-\mathbf{1 3}]$. Some of these works focus on the planar systems in which only one kinematic joint is modelled as a joint clearance. Dubowsky and Freudenstein $[2,3]$ formulated an impact pair model to predict the dynamic response of an elastic mechanical joint with clearance. In their model, springs and dashpots were arranged as Kelvin-Voigt model. This work was subsequently extended by Dubowsky and his coworkers $[14,15]$. Dubowsky [16] showed how clearances can interact dynamically with machine control systems to destabilize and produce undesirable limit cycle behaviour.
Earles and Wu [17] employed a modified Lagrange's equation approach in which constraints were incorporated using Lagrange multipliers in order to predict the behaviour of rigid mechanism with clearance in a journal-bearing. The clearance in the journal-bearing was modelled by a massless imaginary link, but the simulation was restricted to the range of motion that starts when the contact between the journal and the bearing is terminated. Later, they investigated the prediction of contact-loss in a bearing of a linkage mechanism [18]. Mahrus [19] conducted an experimental investigation into journal-bearing performance. A test machine was developed on which steady or varying unidirectional or full twocomponent dynamic load can be applied to the test journal-bearing. The corresponding journal centre path is measured simultaneously with the load to show the effect of the load diagram on hydrodynamic lubrication.

Wilson and Fawcett [20] assumed that clearance exists in the sliding bearing in a slider-crank mechanism in which a theoretical investigation on the effects of parameters, such as the geometry, speed and mass distribution of the mechanism, upon the transverse motion of the slider was reported. They derived the equations of motion for the different scenarios of the slider motion inside the guide. Grant and Fawcett [21] proposed a method to prevent contact-loss between the journal and bearing. Their experimental results verified the approach for a limited class of systems, however, the method is not universal [22]. Townsend and Mansour [23] modelled a four bar crank-rocker mechanism with clearance as two sets of compound pendulums in a theoretical study. They ignore the motion in contact mode entirely, a close succession of small pseudoimpacts was assumed for the simulation. Subsequently, Miedema and Mansour [24] extended their previous two-mode model, for the free flight and impact modes, to a three-mode model in which a following mode was proposed. In their numerical simulations the following mode was always assumed to occur immediately after the impact mode, however, this is frequently not observed in practice $[\mathbf{4}, \mathbf{5}]$.

Haines [25] derived equations of motion that describe the contributions at a revolute joint with clearance but with no lubrication present. Also Haines [11] carried out an experimental investigation on the dynamic behaviour of revolute joints with varying degrees of clearance. Under static loads, the deflection associated with contact elasticity in the dry journal-bearing is found to be much greater and less linear than predicted. Bengisu et al. [26] developed a separation parameter for a four bar linkage, which was based on a zero-clearance analysis. The theoretical results are compared with the experimental results 
and showed a qualitative agreement. They also predicted contact loss in a mechanism with multiple joint clearances.

More recently, Feng et al. [27] developed an optimization method to control the change of inertia forces by optimizing the mass distribution of moving links in planar linkages with clearances at joints. Innocenti [28] presented a method for analysis of spatial structures with revolute joint clearances. Orden [29] presented a methodology for the study of typical smooth joint clearances in mechanical systems. This proposed approach takes advantage of the analytical definition of the material surfaces defining the clearance, resulting in a formulation where the gap does not play a central role, as it happens in standard contact models.

Other researchers also included the influence of the bodies' flexibility in the dynamic performance of mechanical systems besides the existence of gaps in the joints [30-33]. Dubowsky and Moening [15] obtained a reduction in the impact force level by introducing bodies' flexibility. They also observed a significant reduction of the acoustical noise produced by the impacts when the system incorporates flexible bodies. Kakizaki et al. [34] presented a model for spatial dynamics of robotic manipulators with flexible links and joint clearances, where the effect of the clearance is taken to control the robotic system.

The contribution and merit of the present work deals with the quantification of the dynamic response of multi-body systems composed of rigid bodies that include revolute joints with clearance, in which the clearance size and friction coefficient effects are analysed and discussed.

\section{MODELLING OF REVOLUTE JOINTS WITH CLEARANCE}

Figure 1(a) shows the typical configuration of a revolute joint with clearance. The joint elements are the bearing and journal, which diameters are $R_{\mathrm{B}}$ and $R_{\mathrm{J}}$, respectively. The difference between the bearing and journal radii is the radial clearance, $c$. The clearance in realistic joints is much smaller than the size of the bodies; however, in Fig. 1 is grossly exaggerated for illustration. The existence of the clearance in the revolute joints allows two extra degrees of freedom, that is, the horizontal and vertical displacement and, consequently, the journal and bearing can freely move relative to each other. In addition, Fig. 1(a) shows the relative penetration or indentation between the journal and bearing when the two bodies impact to each other.

Three different modes of journal motion inside the bearing are considered, namely the contact or following mode, the free-flight mode, and the impact mode [8]. These three types of the journal motion are, schematically, illustrated in Fig. 1(b). In the contact or following mode, the journal and the bearing are in permanent contact and a sliding motion related to each other is assumed to exist. In this mode the relative penetration depth varies along the circumference of the bearing. Clearly, in practice, this mode is ended when the journal and bearing separate from each other and the journal enters in free flight mode. In the free-flight mode, the journal can move freely inside the bearing boundaries, that is, the journal and the bearing are not in contact and, consequently, no reaction force is developed at the joint. In the impact mode, which occurs at the termination of the free-flight mode, impact forces are applied and removed in the system. This mode is characterized by a discontinuity in the kinematic and dynamic characteristics and a significant exchange of momentum occurs between the two impacting bodies. At the termination of the impact mode, the journal can enter either in free flight or in following mode. During the dynamic simulation of a revolute joint with clearance, if the path of the journal centre is plotted for each instant, these different modes of motion can be easily identified, as shown in Fig. 1(b).

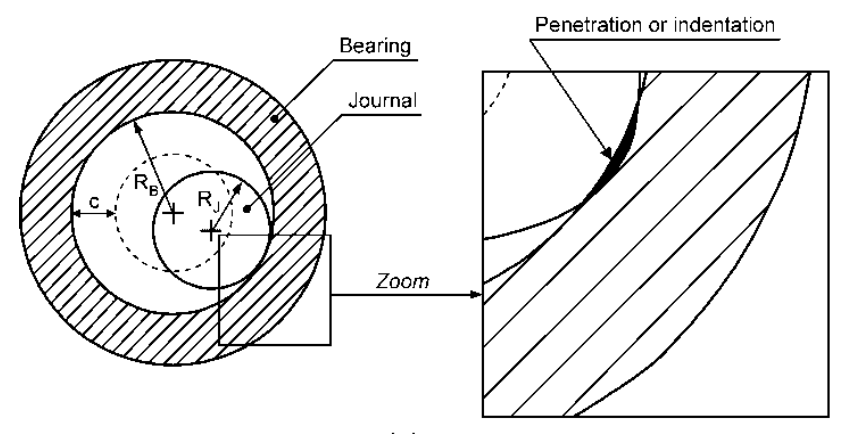

(a)

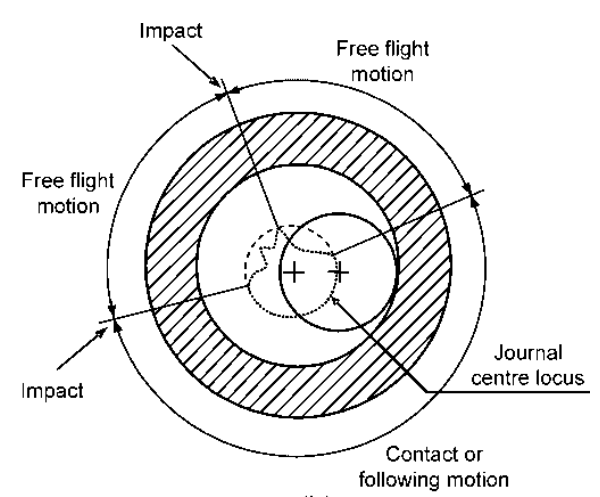

(b)

Fig. 1 (a) Revolute joint with clearance, in which the clearance is exaggerated for clarity and (b) modes of the journal motion inside the bearing boundaries 
The methodology employed in this work assumes that the journal and bearing are modelled as two colliding bodies, being the dynamics of joint controlled by force constraints instead of kinematic constraints as it happens in an ideal revolute joint. Thus, according to this concept, joints with clearance can be called force-joints, as they deal with force constraints. A good detail of the revolute clearance joint formulation can be found in Flores et al. [5].

As it was mentioned, because of the existence of the clearance in the joint, contact forces are generated at the bodies when the journal reaches the bearing wall. In a simple manner, the joint behaviour consists of periods of free-flight motion followed by metal-tometal contact when the journal and bearing collide to each other. In the first situation, there is no reaction force at the joint. In the second case, a contact force between the journal and bearing is developed and the direction of this joint reaction force is parallel to the relative position of the centres of the bearing and the journal, as shown in Fig. 2(a). The joint force magnitude upon the contact can be obtained from the Hertzian contact theory [6], which is based on the assumption that the dimensions of the contact region between the journal and bearing is much smaller than the radius of each body. The contact force model proposed by Lankarani and Nikravesh [6], that accounts for both the elastic and damping effects, is employed in the present work to model evaluated the contact force. The damping effect is associated with the energy dissipated during the impact process, together with the dissipative effect associated with the Coulomb friction on the contact surfaces [7].

The relative penetration or indentation, $\delta$, between the journal and bearing can be written as,

$$
\delta=e-c
$$

where $e$ is the absolute eccentricity and $c$ is the radial clearance.
The eccentricity is given by

$$
e=\sqrt{\Delta X^{2}+\Delta Y^{2}}
$$

in which $\Delta X$ and $\Delta Y$ represent the horizontal and vertical displacements of the journal inside the bearing, respectively. These relative displacements are obtained from the global position vectors of the bearing and journal centres, respectively [8]. Thus, the normal reaction force at the revolute joint clearance can be expressed by $[\mathbf{5}, \mathbf{6}]$

$$
F_{\mathrm{N}}=\left\{\begin{array}{lll}
0 & \text { if } & \delta \leqslant 0 \\
K \delta^{n}\left(1+\frac{3\left(1-c_{e}^{2}\right)}{4} \frac{\dot{\delta}}{\dot{\delta}^{(-)}}\right) & \text {if } & \delta>0
\end{array}\right.
$$

where $K$ generalized stiffness coefficient, $\delta$ is the relative penetration, $c_{e}$ is the restitution coefficient, $\dot{\delta}$ is the relative penetration velocity, and $\dot{\delta}^{(-)}$is the initial impact velocity. The exponent $n$ is equal to 1.5 for metallic contacts. For two spheres in contact the generalized stiffness coefficient is function of the radii of the spheres $i$ and $j$ and the material properties as [35]

$$
K=\frac{4}{3\left(\sigma_{i}+\sigma_{j}\right)} \sqrt{\frac{R_{i} R_{j}}{R_{i}+R_{j}}}
$$

where the material parameters $\sigma_{i}$ and $\sigma_{j}$ are given by

$$
\sigma_{k}=\frac{1-v_{z}^{2}}{E_{z}} \quad(z=i, j)
$$

variables $v_{z}$ and $E_{z}$ are, respectively, the Poisson's ration and the Young's modulus of elasticity associated with the material of each sphere.

The contact force model developed by Lankarani and Nikravesh [6] uses the general trend of the Hertz contact law, in which a hysteresis damping function is incorporated with the intent to represent the

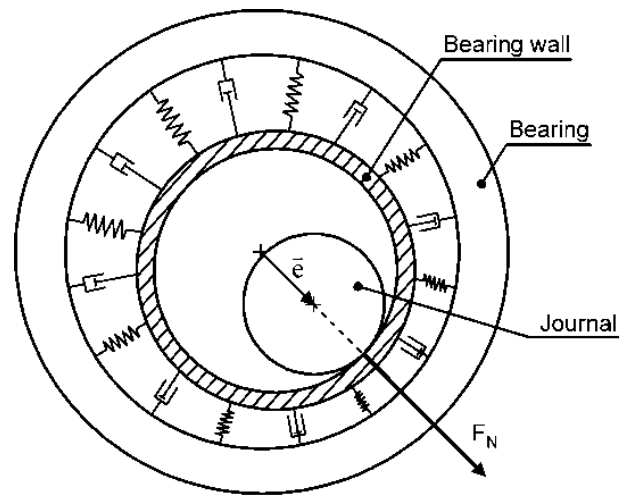

(a)

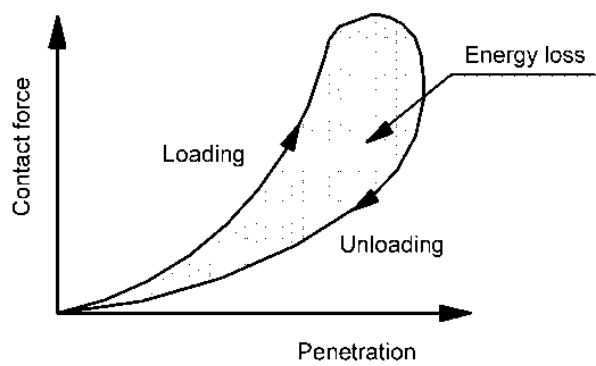

(b)

Fig. 2 (a) Contact between the journal and bearing represented by a non-linear viscous-elastic force model and (b) contact force versus penetration depth 
energy dissipated during the impact. In fact, when an elastic body is subjected to cyclic load, the energy loss in the material causes a hysteresis loop in the force-penetration relation. This contact model is only valid for impact velocities lower than the propagation velocity of elastic waves across the bodies. The use of the damping scheme included in this model implies the outcome illustrated in Figs 3(a) to (c) in which the penetration depth, $\delta$, normal contact force, $F_{\mathrm{N}}$, and hysteresis of an impact between two internally spheres are presented. The generalized stiffness coefficient used is $6.6 \times 10^{10} \mathrm{~N} / \mathrm{m}^{1.5}$. Figures $4(\mathrm{a})$ and (b) show the time variation of the contact force and the relation between the force and penetration depth for different values of the coefficient of restitution. Observing Fig. 4(b), it is important to note how the hysteresis loop increases when the coefficient of restitution decreases. When the restitution coefficient is equal to 1.0 , which corresponds to the pure Hertz contact law there is no energy dissipation in contact. This observation is evident in the force-penetration depth relation of Fig. 4(b), which does not present hysteresis loop.

In simulating the dynamics of mechanical systems with clearance joints, it is essential to determine the instant of contact between the bodies that constitute the joint. The coupling of the relatively slow motion of the overall system with the faster motion, associated with the joint clearance parameters, makes the eigenvalues of the matrix of coefficients and independent terms of the equations of motion widely spread. Hence, numerical algorithms with variable step size and order are an important feature for the computational strategy [36]. The use of these numerical schemes plays a key role in contact problems, whose dynamic response is quite complex and discontinuous, because of the sudden change in kinematic structure caused by rapid variation of the contact forces applied to the system and to the dramatic change in the system stiffness, when a contact condition is achieved. Furthermore, the numerical model to characterize the contact between the bodies requires the knowledge of the preimpact conditions, that is, the impact velocity and the direction of the plane of collision [5]. The contact duration, as well as the penetration depth, cannot be predicted from the preimpact conditions because of the influence of the kinematic constraint imposed by all bodies in the overall system motion. Thus, before the first impact, the joint elements can freely move relative to each other and, in this phase, the step size is relatively large and the global configuration of the system is characterized by large translational and rotational displacements. Therefore, the first impact between

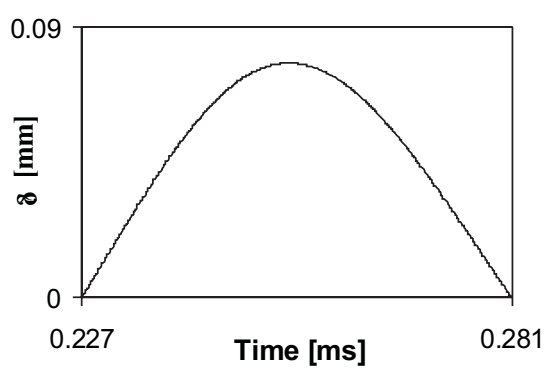

(a)

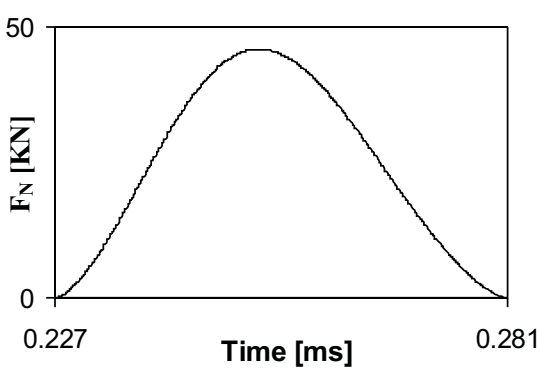

(b)

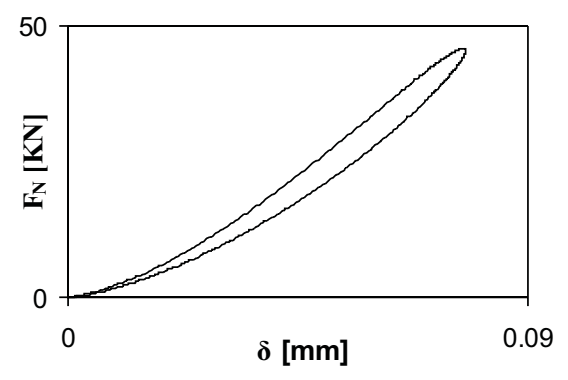

(c)

Fig.3 Internally colliding spheres modelled by Lankarani and Nikravesh contact force model: (a) penetration depth, $\delta$; (b) normal contact force, $F_{\mathrm{N}}$; (c) force-penetration relation

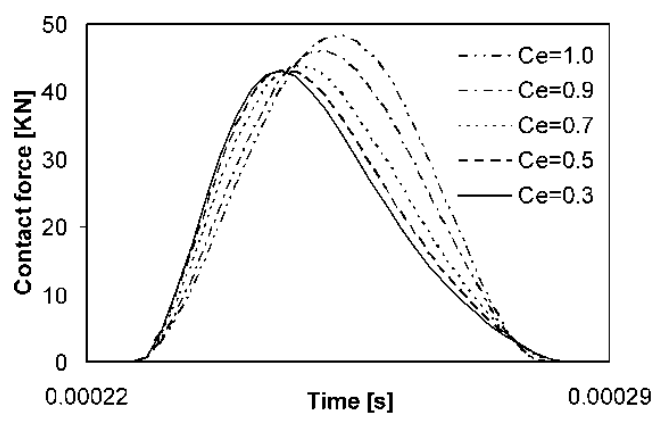

(a)

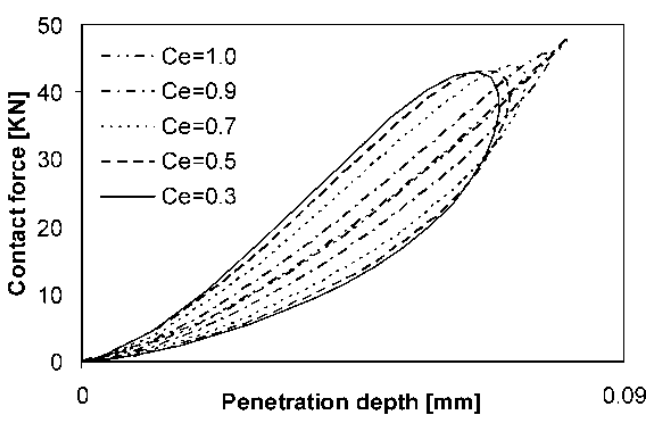

(b)

Fig. 4 Influence of the coefficient of restitution for the Lankarani and Nikravesh continuous contact force model: (a) contact force versus time and (b) force-penetration depth relation 
the colliding bodies is often made with a high penetration depth, and, consequently, the contact forces are large too. This forces the integration process to go back and take a smaller step size, until a step can be taken which is within the given error tolerance.

The journal is considered in free-flight motion relative to the bearing until the geometric equality criterion of equation (1) is verified, and, consequently, the contact between the journal and bearing wall is initialized. Ideally, when $\delta=e-c=0$, the bearing and the journal are in contact to each other. However, because of the accumulation of computational round-off errors, a tolerance is introduced in order to accommodate for inaccuracies in the numerical results. In the present work, the bearing and journal are considered to be in contact when the penetration depth is larger than $1.0 \times 10^{-10} \mathrm{~m}$. Therefore, when the 'first' penetration is within the penetration tolerance it is assumed that such is the moment of the impact and the position and relative velocity of the contact points and the direction of the plane of collision are recorded. It should be highlighted that with this methodology, the step size can reach smaller values than those needed to keep the integration tolerance error under control. When the step size goes below the limit, it is forced to remain at the minimum value [8].

In short, in the present work, the journal and the bearing are elements that constitute a revolute joint clearance and they are treated as impacting bodies. Depending on the relative positions of the centres of the journal and bearing during the mechanism motion, the presented methodology can determine whether or not the contact at the joints occurs. If it does, contact forces are evaluated and applied to the journal and bearing. If not, no reaction forces at the joint are developed. The contact forces developed at the joint clearances due to the impact are evaluated as non-linear function of the local deformation between the colliding bodies, as well as function of the geometric and material properties of the contacting surfaces. The contact forces are introduced into the equations of motion as external forces.

\section{EQUATIONS OF MOTION FOR A CONSTRAINED MULTI-BODY SYSTEM}

In this section, the equations of motion for a general mechanical system are presented [36]. The algebraic kinematic independent holonomic constraints that describe the configuration of a multi-body system can written as

$$
\Phi=(\boldsymbol{q}, t)
$$

where $\boldsymbol{q}$ is the vector of generalized coordinates that describes the configuration of all bodies that constitute the mechanical system and $t$ is the time variable.
The equations of motion for a mechanical system can be represented by [36]

$$
\mathbf{M} \ddot{\boldsymbol{q}}+\boldsymbol{\Phi}_{q}^{\mathrm{T}} \lambda=\boldsymbol{g}
$$

where $\mathbf{M}$ is the generalized mass matrix, $\ddot{\boldsymbol{q}}$ is the vector that contains the system accelerations, $\boldsymbol{\Phi}_{\mathbf{q}}$ is the Jacobian matrix, $\lambda$ is the Lagrange multipliers vector, and $\boldsymbol{g}$ is the generalized force vector.

In dynamic analysis, a unique solution is obtained when the constraint equations are considered simultaneously with the differential equations of motion, for a proper set of initial conditions, consequently, the constraint velocity and acceleration equations are required. Indeed, there are methods capable of solving differential algebraic equations (DAEs) without differentiating the constraint equations [36]. Thus, differentiating equation (6) with respect to time yields the velocity constraint equations

$$
\boldsymbol{\Phi}_{\mathbf{q}} \dot{\boldsymbol{q}}=-\boldsymbol{\Phi}_{t} \equiv v
$$

in which $\dot{\boldsymbol{q}}$ is the vector of generalized velocities and $v$ is the right-hand side of velocity equations, which contains the partial derivates of $\boldsymbol{\Phi}$ with respect to time, $\partial \Phi / \partial t$. It should be noticed that only rheonomic constraints, associated with driver equations, contribute with non-zero entries to the vector $v$.

A second differentiation of equation (6) with respect to time leads to the acceleration constraint equations

$$
\boldsymbol{\Phi}_{\mathrm{q}} \ddot{\boldsymbol{q}}=-\left(\boldsymbol{\Phi}_{\mathbf{q}} \dot{\boldsymbol{q}}\right)_{\mathbf{q}} \dot{\boldsymbol{q}}-2 \boldsymbol{\Phi}_{\mathbf{q} t} \dot{\boldsymbol{q}}-\boldsymbol{\Phi}_{t t} \equiv \boldsymbol{\gamma}
$$

where $\ddot{\boldsymbol{q}}$ is the acceleration vector and $\boldsymbol{\gamma}$ is the right hand side of acceleration equations, i.e. the vector of quadratic velocity terms, which contains the terms that are exclusively function of velocity, position and time. In the case of holonomic scleronomic constraints, that is, when $\boldsymbol{\Phi}$ is not explicitly dependent on the time, the term $\boldsymbol{\Phi}_{t}$ in equation (8) and the $\boldsymbol{\Phi}_{\mathbf{q} t}$ and $\boldsymbol{\Phi}_{t t}$ terms in equation (9) vanish. Equations (6), (8), and (9) must be satisfied during the numerical simulation. Equation (9) can be appended to equation (7) and rewritten in matrix form as

$$
\left[\begin{array}{cc}
\mathbf{M} & \boldsymbol{\Phi}_{\mathbf{q}}^{\mathrm{T}} \\
\boldsymbol{\Phi}_{\mathbf{q}} & 0
\end{array}\right]\left\{\begin{array}{l}
\ddot{\boldsymbol{q}} \\
\lambda
\end{array}\right\}=\left\{\begin{array}{l}
\boldsymbol{g} \\
\boldsymbol{\gamma}
\end{array}\right\}
$$

This system of equations is solved for $\ddot{\boldsymbol{q}}$ and $\lambda$. Then, in each integration time step, the accelerations vector, $\ddot{\boldsymbol{q}}$, together with velocities vector, $\dot{\boldsymbol{q}}$, are integrated in order to obtain the system velocities and positions for the next time step. This procedure is repeated up to final time analysis is reached [36]. In order to keep the constraint violations under control, the Baumgarte stabilization method is used [37]. Furthermore, the integration process is performed using a predictorcorrector algorithm with variable order and step, such the one suggestion by Shampine and Gordon [38]. 


\section{POINCARÉ MAPS: PERIODIC OR CHAOTIC BEHAVIOUR}

It is not surprising that non-linear behaviour can occur widely in mechanical systems, particularly since all have some inherent non-linearity. In multibody mechanical systems non-linearities arise from intermittent motion, clearance joints, friction effect, and contact-impact forces among others.

Dynamical systems are often used to describe a set of variables with change over time. Mathematically, a dynamical system is described by an initial value problem. When the behaviour of the system is quite sensitive to initial conditions the system is called chaotic. Indeed, the fundamental characteristic of a chaotic physical system is its extreme sensitivity to the initial sate. Sensitivity means that if two identical mechanical systems are started at initial conditions $y$ and $y+\varepsilon$, respectively, where $\varepsilon$ is a very small quantity, their dynamical states will diverge from each other quickly. Sensitive dependence does not require exponential growth of perturbation but this is typical for chaotic systems.

Poincaré maps are considered to be the most successful tool for the investigation of dynamical systems in theory as well as in applications. Poincaré maps are mathematical abstractions which are often useful in highlighting the behaviour of systems in terms of periodic, quasi-periodic, and chaotic, or non-linear motion. Especially chaotic systems are often examined through the use of Poincaré maps. A Poincaré map consists of plotting the value of two components from the state vector versus its derivative, i.e. $y(t)$ and $\dot{\boldsymbol{y}}(t)[39,40]$.

Regular or periodic behaviour is insensitive to initial conditions and is represented in the Poincaré map by a closed orbit or finite number of points. When the periodic response becomes unstable the typical result will be a geometrically strange object, called strange attractor, which is a strong indicator of chaotic behaviour. The chaotic motion is called Hamilton when the Poincaré map points will spread all over the map, that is, as the simulation time marches on, more and more points will be added to the map. Chaotic or non-periodic responses are extremely sensitive to initial conditions and are densely filled by orbits or points in the Poincaré map. Indeed, a complicated looking phase in a Poincaré map is one indicator of chaotic motion. Quasi-periodic orbits fill up the Poincare maps as the chaotic orbits, but they do so in a fully predictable manner since there is no sensitive dependence on the initial conditions [41].

In the present work, Poincaré maps are used to illustrate the dynamic behaviour of planar rigid multibody systems with revolute joints with clearance and friction.

\section{DEMONSTRATIVE EXAMPLE 1: FOUR-BAR MECHANISM}

In this section, the academic four-bar mechanism is used as an illustrative example to demonstrate how a revolute joint with clearance affect a mechanism behaviour. The four-bar mechanism consists of four rigid bodies that represent the ground, crank, coupler, and follower. The body numbers and their corresponding local coordinate systems are shown in Fig. 5. The joints of this mechanism include three ideal revolute joints connecting the ground to the crank, the crank to the coupler, and the ground to the follower. A revolute joint with clearance exists between the coupler and follower. Figure 5 shows three frames from an animation sequence of the four bar mechanism with a joint clearance, in which the clearance is strongly exaggerated to demonstrate the bouncing effect.
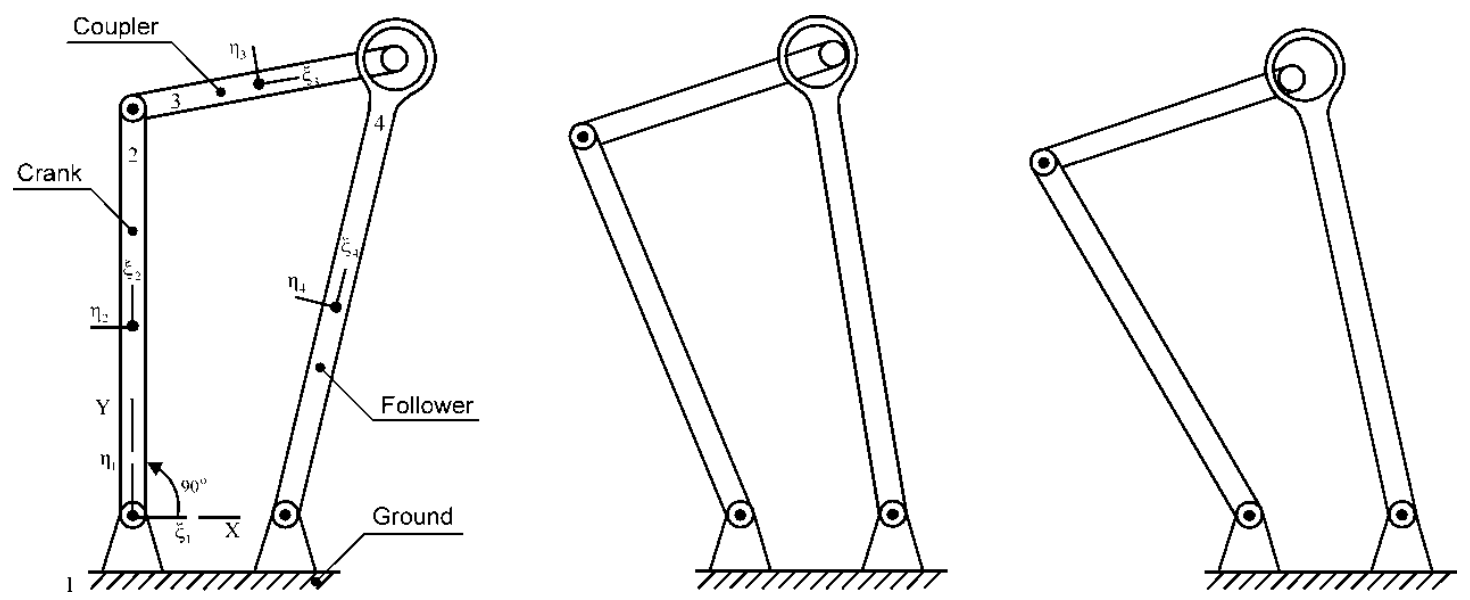

Fig. 5 Four-bar mechanism with a revolute joint clearance between the coupler and follower. The left-most picture corresponds to the initial simulation configuration 
Table 1 Geometric and inertia properties of the four-bar mechanism

\begin{tabular}{llll}
\hline Body nr. & Length $(\mathrm{m})$ & Mass $(\mathrm{kg})$ & Moment of inertia $\left(\mathrm{kg} \mathrm{m}^{2}\right)$ \\
\hline 1 & 0.150 & - & - \\
2 & 0.400 & 3.120 & 0.04225 \\
3 & 0.260 & 2.028 & 0.01185 \\
4 & 0.460 & 3.588 & 0.06402 \\
\hline
\end{tabular}

Table 2 Parameters used in the dynamic simulation of the four-bar mechanism

\begin{tabular}{llll}
\hline Bearing radius & $10.0 \mathrm{~mm}$ & Young's modulus & $207 \mathrm{GPa}$ \\
Journal radius & $9.8 \mathrm{~mm}$ & Poisson's ratio & 0.3 \\
Radial clearance & $0.2 \mathrm{~mm}$ & Integration step size & $0.00001 \mathrm{~s}$ \\
Restitution coefficient & 0.9 & Integration tolerance & 0.000001 \\
\hline
\end{tabular}

The dimensions and inertia properties of each body are listed in Table 1 . The dynamic parameters, used for the simulation and for the numerical methods required to solve the system dynamics, are listed in Table 2.

In order to initiate the numerical simulation, a set of initial conditions is required and the selection of these appropriate conditions play a crucial role in the prediction of the dynamical response of the mechanical systems. The initial conditions for the methodology used here are based on the results of the kinematic analysis of the four bar mechanism, in which all the joints are assumed to be ideal or perfect. The subsequent initial conditions for each time step in the simulation are obtained from the final conditions of the previous time step. This process is performed until the final time of analysis is reached. The crank is the driving link and rotates at a constant angular velocity of $50 \pi \mathrm{rad} / \mathrm{s}$.

Two different kinds of results are presented. Firstly, the four-bar mechanism is modelled considering all the joints as ideal, in which neither clearance nor friction exists and the rotating axes of the journal and bearing remained aligned. Secondly, the mechanism is simulated with a revolute joint clearance, which leads to surface contact in the links. The dynamic response of the four-bar mechanism is obtained and represented in Figs 6(a) and (b) by the time plots of the velocity and acceleration of the follower. The moment acting on the crank, which is required to maintain the crank angular velocity constant, is plotted in Fig. 6(c). The relative motion between the journal and bearing centres is illustrated in Fig. 6(d). The Hertz contact force law with hysteretic damping factor is used to evaluate the contact force between the journal and bearing. Note that the results are plotted against those obtained for ideal joint, being reported for the two full crank rotations after steady-state has been reached.

In general, the angular velocity of the follower is not affected by the existence of the joint clearance, as illustrated in Fig. 6(a). Indeed, there is no deviation in the follower velocity curve when the four-bar mechanism is simulated with ideal and clearance joints. In sharp contrast to the follower angular velocity, the angular acceleration of the follower presents significant differences between the dynamic response of the four-bar mechanism when modelled with and without joint clearance. Upon reviewing Fig. 6(b), it is clear that the acceleration peaks are directly related to the contact between the journal and bearing during the simulation. These peaks are propagated throughout the rigid bodies until the crank moment diagram, in which some deviation from the ideal curve is also visible, as shown in Fig. 6(c). Furthermore, the system's response clearly repeats itself from cycle to cycle. From Fig. 6(d) it is evident that the journal is always in contact with the bearing wall. This observation is logical since the two bodies are moving in the same direction.

In order to better understand the dynamic effect of the joint clearance on the response of the four-bar mechanism, two dimensionless amplification parameters are defined for follower angular acceleration and for the crank moment. The amplification parameter for the follower angular acceleration is expressed by

$$
\operatorname{AP}(\alpha)=\frac{\alpha_{\mathrm{c}}-\alpha_{\mathrm{i}}}{\alpha_{\mathrm{i}}} \times 100
$$

where $\alpha_{\mathrm{c}}$ and $\alpha_{\mathrm{i}}$ are the maximum angular acceleration of the follower of the system with joint clearance and ideal joint, respectively. Similarly, the amplification parameter for crank moment can be written as

$$
\mathrm{AP}(M)=\frac{M_{\mathrm{c}}-M_{\mathrm{i}}}{M_{\mathrm{i}}} \times 100
$$

in which $M_{\mathrm{c}}$ and $M_{\mathrm{i}}$ are the maximum crank moment of the system with joint clearance and ideal joint, respectively. Extracting the maximum values of the follower acceleration and crank moment from Figs 6(b) and (c), the amplification parameters obtained are 50.82 and 38.32 per cent, respectively. These values represent how the level of follower angular acceleration and crank moment is increased because of the contact within the joint clearance. In short, the joint clearance creates significantly larger dynamic accelerations and reaction moments on the mechanism than those obtained for an ideal dynamic model. Furthermore, the results have to be understood under the assumptions adopted in this work. Indeed, currently our model does not account for bodies' flexibility, which is a strong factor that affects/reduces the high peaks on the system output results. Certainly that if the bodies' elasticity is taken into account and structural damping also, these peaks should be reduced. Finally, notice that many of 


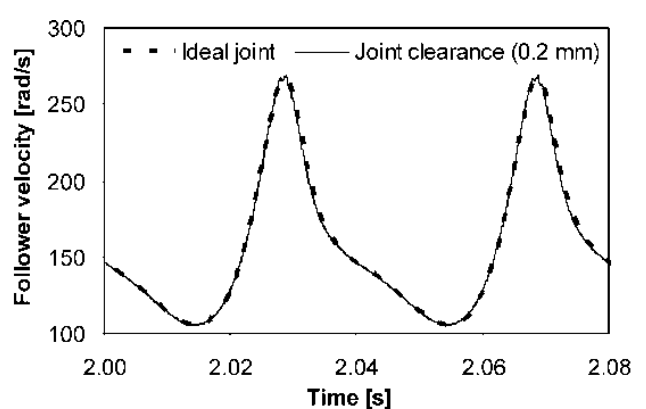

(a)

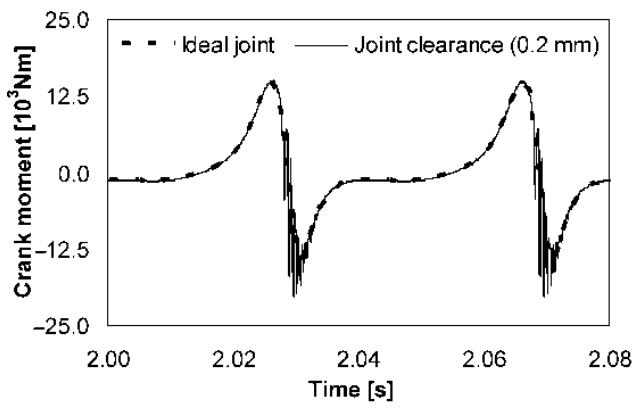

(c)

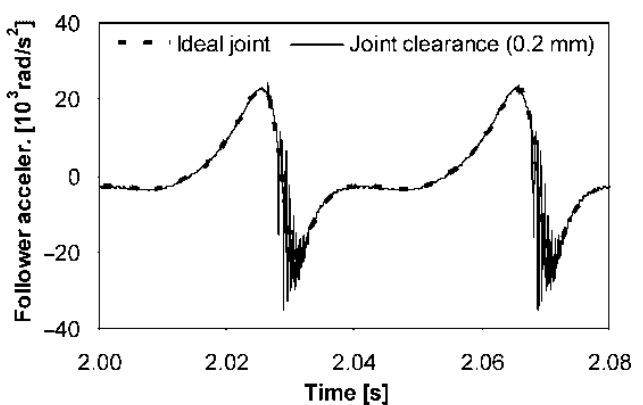

(b)

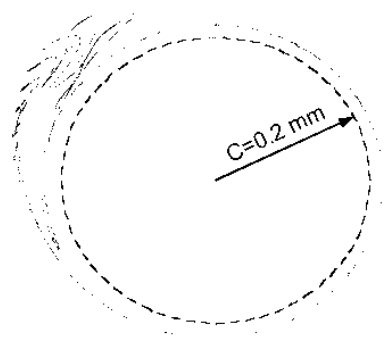

(d)

Fig. 6 (a) Follower angular velocity; (b) follower angular acceleration; (c) crank moment required to maintain the crank angular velocity constant; (d) journal centre path relative to the bearing centre

the results published in literature when dealing with accelerations, are filtered, which is not the case here.

Figure 7 shows the influence of the radial clearance size on the crank reaction moment. The values of the

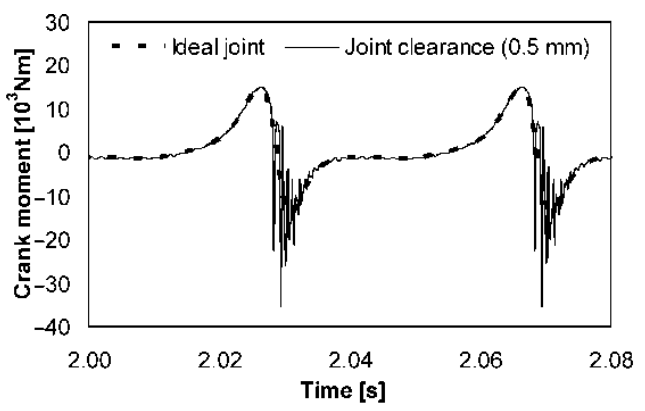

(a)

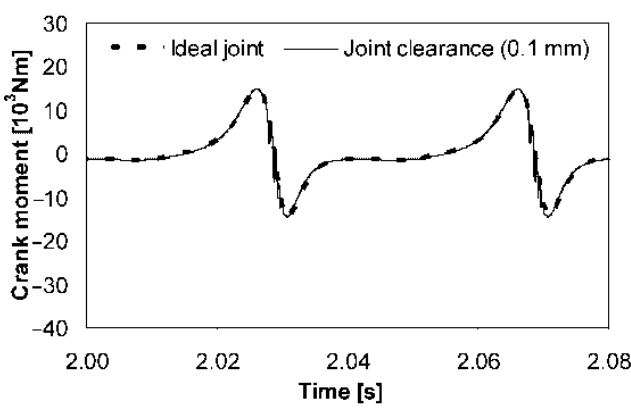

(c) radial clearance used are $0.5,0.2,0.1$, and $0.01 \mathrm{~mm}$. The crank moment diagrams tend to be close to those obtained for ideal joint when the clearance size is reduced. The same conclusion can be drawn from

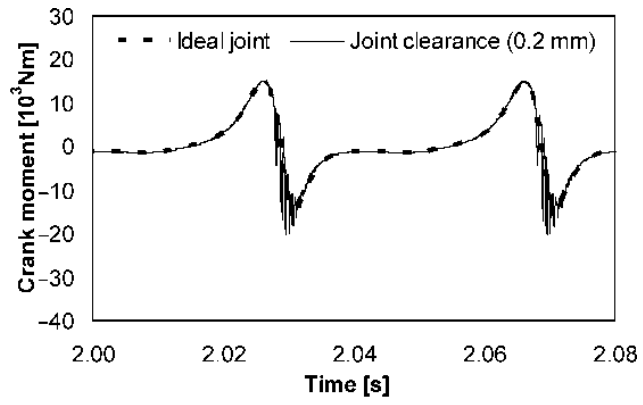

(b)

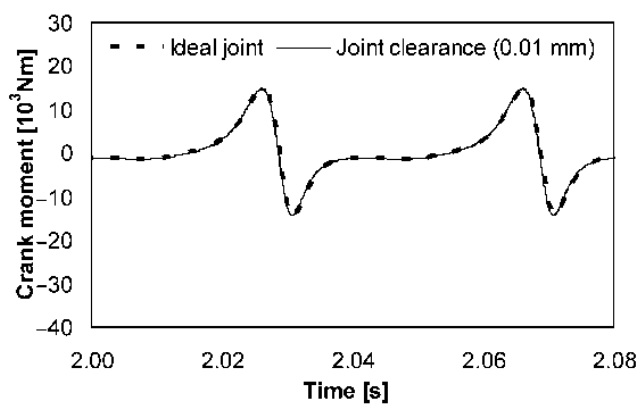

(d)

Fig. 7 Crank moment for various radial clearances: (a) $c=0.5 \mathrm{~mm}$; (b) $c=0.2 \mathrm{~mm}$; (c) $c=0.1 \mathrm{~mm}$; (d) $c=0.01 \mathrm{~mm}$ 


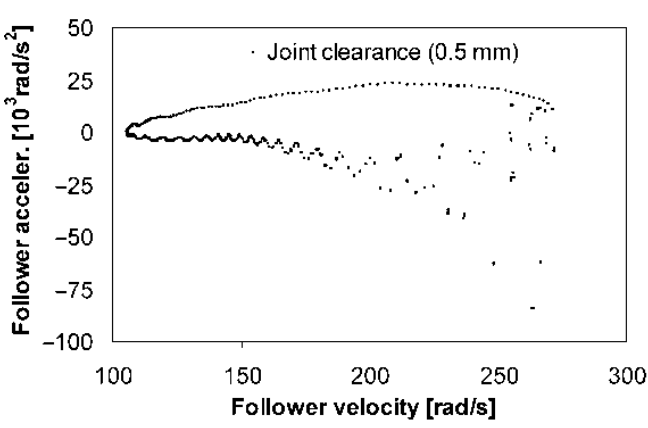

(a)

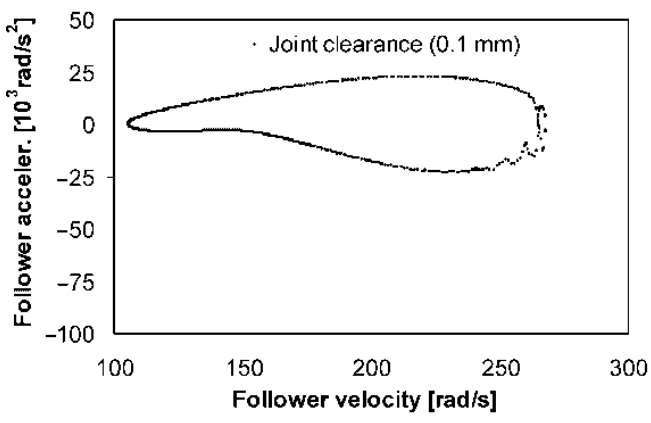

(c)

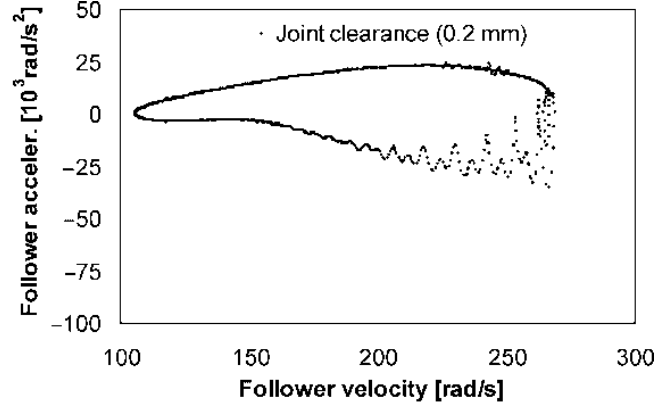

(b)

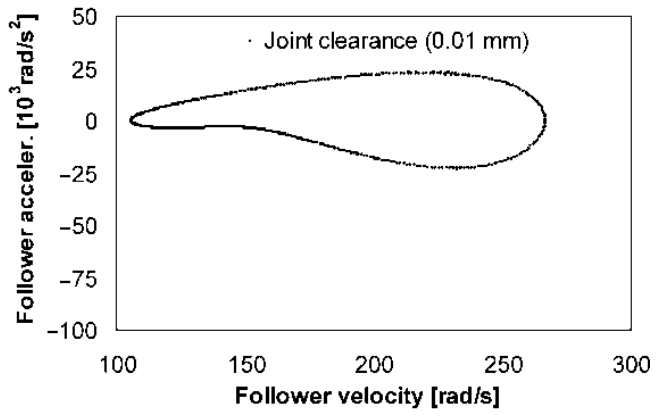

(d)

Fig. 8 Poincaré map for various radial clearances: (a) $c=0.5 \mathrm{~mm}$; (b) $c=0.2 \mathrm{~mm}$; (c) $c=0.1 \mathrm{~mm}$; (d) $c=0.01 \mathrm{~mm}$

Fig. 8 where the Poincaré maps are used to illustrate the dynamic behaviour of the four-bar mechanism. When a joint clearance is included in the mechanism, the systems' response tends to a periodic or non-linear instead of regular or periodic. In fact, the Poincaré map for the case simulation with a joint clearance presents a more complex aspect in an open orbit form, which is a strong indicator of non-linear behaviour. The degree of non-linearity decreases with the radial clearance size. This feature can be useful in calculations of the acceptable range for clearance for any type of construction where this type of joint clearance is applied.

\section{DEMONSTRATIVE EXAMPLE 2: SLIDER-CRANK MECHANISM}

In this section, the slider-crank mechanism is used to illustrate the dynamic behaviour of the revolute clearance joints in multi-body systems. Figure 9 depicts the kinematic configuration of the slider-crank mechanism, which consists of four bodies, including ground, two ideal revolute joints, and one ideal translational joint. A revolute clearance joint exists between the connecting rod and slider. The body numbers and their corresponding coordinate systems are shown in Fig. 9.

In order to keep the analysis simple and to illustrate the dynamic clearance joint behaviour, all the bodies are considered to be rigid and the inertia due to

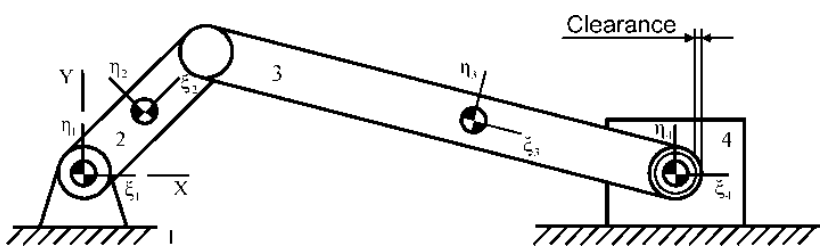

Fig. 9 Slider-crank mechanism with a clearance joint

the driving motor is neglected. The length and inertia properties of each body are shown in Table 3 .

In the dynamic simulation the crank is the driving body and rotates with a constant angular velocity equal to $5000 \mathrm{r} / \mathrm{min}$ clockwise. The initial configuration corresponds to crank and connecting rod collinear and the position and velocity journal centre are taken to be zero. Initially, the journal and bearing centres are coincident. Table 4 shows the dynamic parameters used in simulations.

In order to analyse the dynamic behaviour of the system, long time simulations were performed and the results presented below are relative to two full

Table 3 Geometric and inertia properties of the slider-crank mechanism

\begin{tabular}{llll}
\hline Body nr. & Length $(\mathrm{m})$ & Mass $(\mathrm{kg})$ & Moment of inertia $\left(\mathrm{kg} \mathrm{m}^{2}\right)$ \\
\hline 2 & 0.050 & 0.300 & 0.00001 \\
3 & 0.120 & 0.210 & 0.00025 \\
4 & - & 0.140 & - \\
\hline
\end{tabular}


Table 4 Parameters used in the dynamic simulation of the slider-crank mechanism

\begin{tabular}{llll}
\hline Bearing radius & $10.0 \mathrm{~mm}$ & Poisson's ratio & 0.3 \\
Restitution coefficient & 0.9 & Baumgarte $-\alpha, \beta$ & 5 \\
Friction coefficient & 0.0 & Integration step size & $0.00001 \mathrm{~s}$ \\
Young's modulus & $207 \mathrm{GPa}$ & Integration tolerance & 0.00001 \\
\hline
\end{tabular}

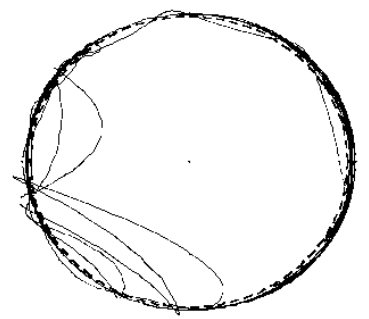

(a)

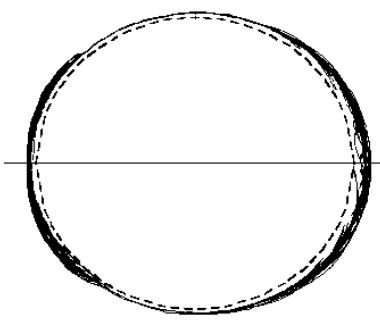

(c)

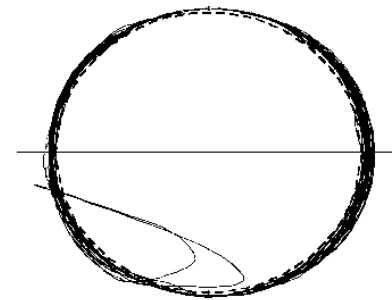

(b)

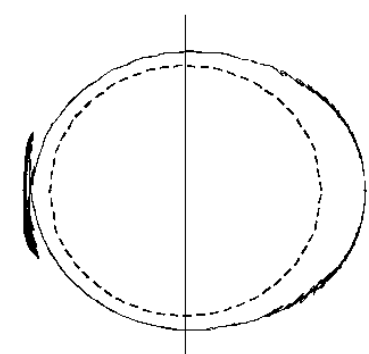

(d)
Fig. 10 Journal centre orbit for different clearance sizes: (a) $c=0.5 \mathrm{~mm}$; (b) $c=0.2 \mathrm{~mm}$; (c) $c=0.1 \mathrm{~mm}$; and (d) $c=0.01 \mathrm{~mm}$

crank rotations after steady-state has been reached. The revolute clearance joint is modelled as dry impact between the two bodies that constitute the journal bearing element. When contact is detected, together with the penetration depth and surface properties, the normal contact force is evaluated by equation (3). The tangential or friction force is modelled according to a modified Coulomb law [7].

The behaviour of the revolute clearance joint is illustrated by plotting the trajectory of the journal inside the bearing boundaries as well as the plot of Poincaré maps. In the present example, the slider velocity and the slider acceleration are chosen to plot the Poincaré maps. The effect of the radial clearance size and friction effect are studied separately. In the first case the influence of the radial size clearance is performed. The range of the clearance used in the present work is $0.5,0.2,0.1$, and $0.01 \mathrm{~mm}$. Figures 10 (a) to (d) show the journal centre trajectory inside the bearing. The Poincaré maps are illustrated through Figs 11(a) to (d).

Figure 10(a) shows a non-linear motion between the journal and bearing since the motion does not repeat from cycle to cycle. This is confirmed by the respective Poincaré map, see Fig. 11(a), where chaotic behaviour can clearly be observed. The chaotic response suggests that impacts followed by some rebounds take place. Figure 11(b) shows a quasi-periodic motion, because the orbits fill up the Poincaré maps in a fully predictable manner since there is no sensitive dependence on the initial conditions. It is clear that when the clearance is reduced the dynamic behaviour tends to be periodic or regular, which indicates that the journal follows the bearing wall. Indeed, the close orbit in the Poincaré map of Fig. 11(d) is clearly a periodic response. It should be noted that for an ideal joint the Poincaré map is almost the same as the map shown in Fig. 11(d), which is expected in so far as all the bodies have periodic motion and repeated every crank cycle. From the Poincaré maps analysis, the journal centre path is characterized and it is possible to distinguish between periodic, quasi-periodic and chaotic responses.

In the second simulation of the slider-crank mechanism, the friction phenomenon is included in the dynamics of the revolute clearance joint. The radial clearance size is equal to $0.5 \mathrm{~mm}$ and four different values for friction coefficient are used, namely, $0.01,0.03,0.05$, and 0.10 . The journal centre trajectories inside the bearing boundaries are illustrated in Figs 12(a) to (d). The corresponding Poincaré maps which relate the slider acceleration versus slider velocity are shown in Figs 13(a) to (d).

Looking at the Figs 12(a) to (d) and 13(a) to (d) it is clear that, when the friction coefficient increases, the dynamic response of the system tends to be simply periodic. For low value of friction coefficient, the system response is chaotic since the Poincaré map has a spread of points. However, for higher values, the system changes from chaotic to quasi-periodic and even regular motion because the orbits on the Poincaré maps tend to be close orbits in a rather complicated manner. Broadly, observing Figs 12(a) to (d) and 13(a) to (d) it can be concluded that the increase of friction coefficient leads to a better response of the system, that is, transition from chaotic or non-linear motion to periodic or regular motion.

In short, multi-body systems with clearance joints are well-known as non-linear dynamic systems, under certain conditions exhibit chaotic response, which is typically characterized by non-periodic response and extreme sensitivity to initial conditions. Moreover, when the friction effect is included, the degree of non-linearity increases. However, from the results presented in this section, it is found that the dynamics of the revolute clearance joint in rigid multi-body systems is quite sensitive to the clearance size as well as to the friction coefficient, and with only small change in one of these parameters, the response of the system can shift from chaotic to periodic. 


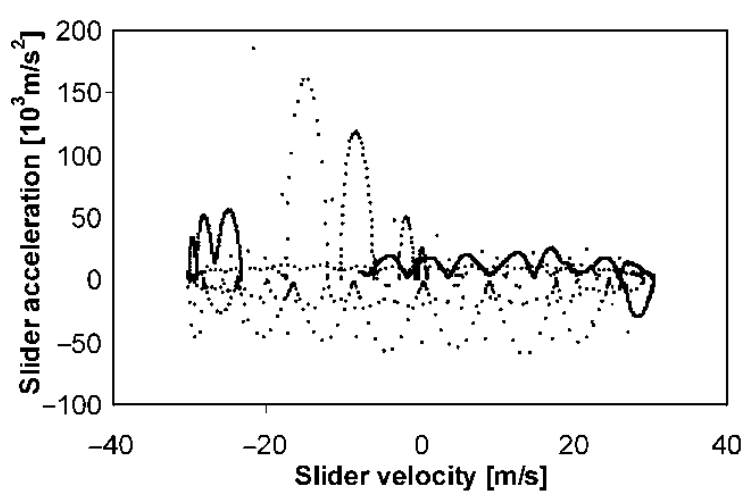

(a)

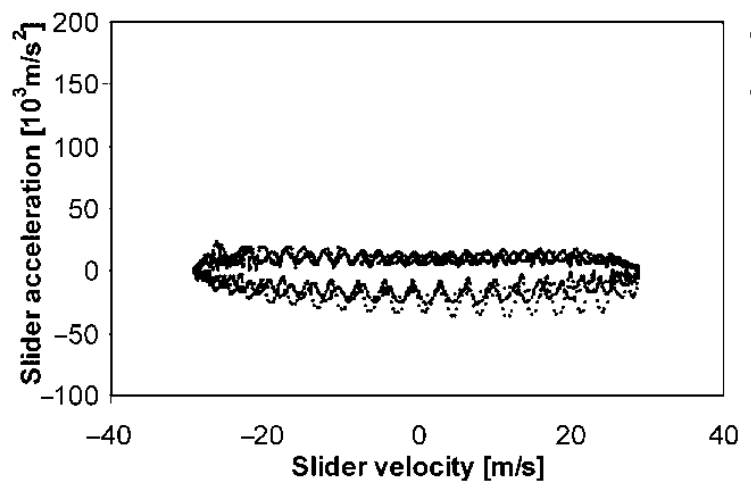

(c)

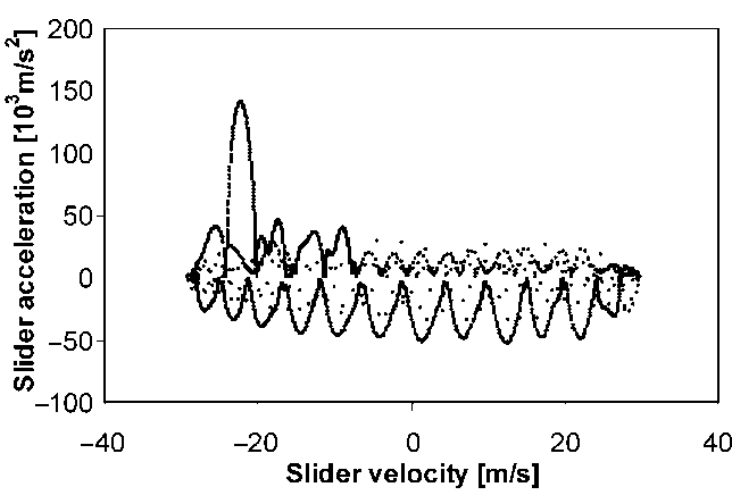

(b)

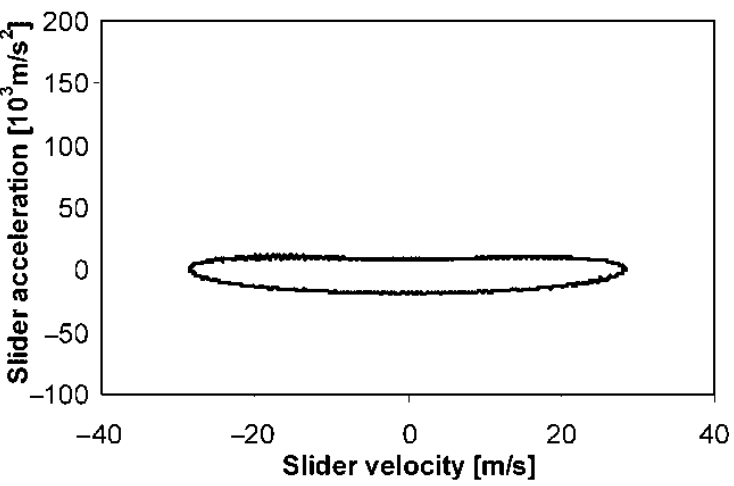

(d)

Fig. 11 Poincaré maps for different clearance sizes: (a) $c=0.5 \mathrm{~mm}$; (b) $c=0.2 \mathrm{~mm}$; (c) $c=0.1 \mathrm{~mm}$; (d) $c=0.01 \mathrm{~mm}$

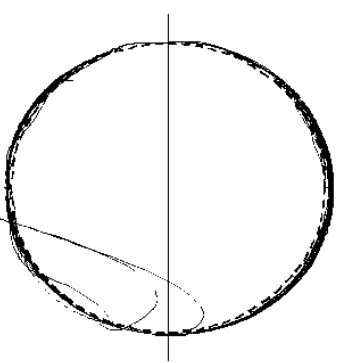

(a)

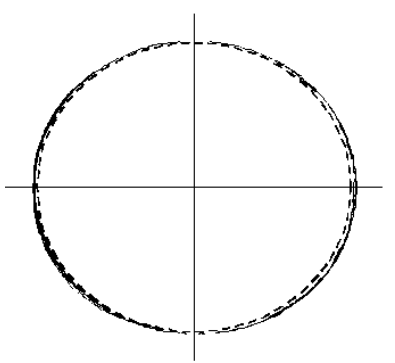

(c)

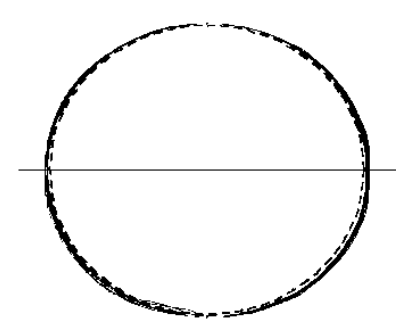

(b)

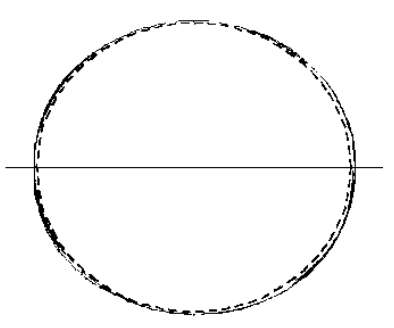

(d)
Fig. 12 Journal centre orbit for different friction coefficients: (a) $c_{\mathrm{f}}=0.01$; (b) $c_{\mathrm{f}}=0.03$; (c) $c_{\mathrm{f}}=0.05$; (d) $c_{\mathrm{f}}=0.10$

\section{CONCLUSIONS}

The application of existing techniques for modelling of impacts in multi-body systems was presented and discussed in this paper. In particular, the influence of the clearance size and the friction coefficient on the dynamic response of planar rigid multi-body systems including revolute joints with clearance was studied. Numerical results for two simple planar mechanisms with revolute clearance joints were used to demonstrate the methodology and assumptions adopted throughout this work.

For the radial clearance sizes and friction coefficient values used in this work the trajectory of the journal inside the bearing exhibits both periodic and chaotic responses. This is confirmed through the use of the Poincaré maps. When the clearance is reduced, the dynamic response changes from chaotic to periodic or regular behaviour. The same conclusion is drawn when the friction coefficient is increased. Indeed, the clearance size and friction coefficient act in a similar way. The dynamic response, in terms of journal centre trajectory and Poincaré maps, shows that the periodic and non-periodic response can occur. Poincaré maps play a key role in representing and understanding the global behaviour of dynamical systems. This feature can be useful in calculation of the acceptable range for clearance during the design process. 


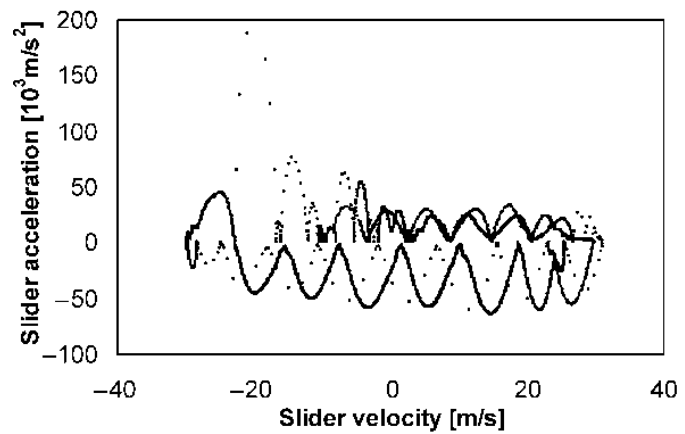

(a)

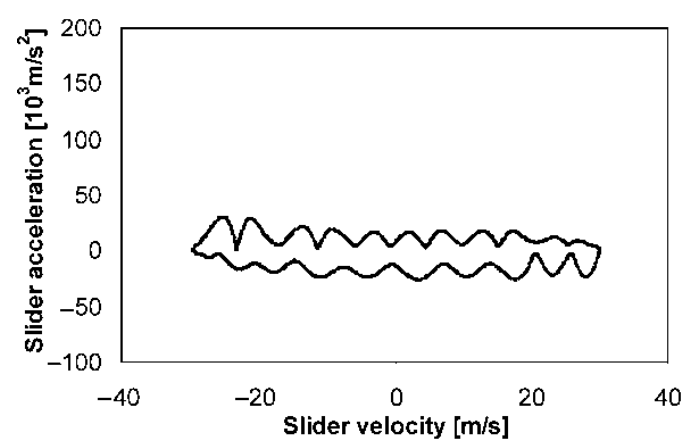

(c)

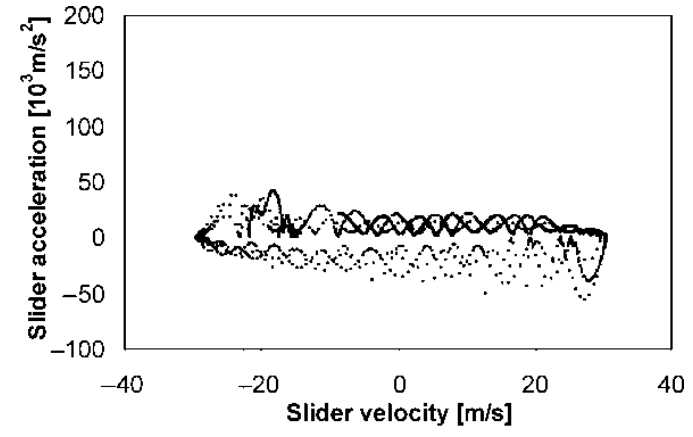

(b)

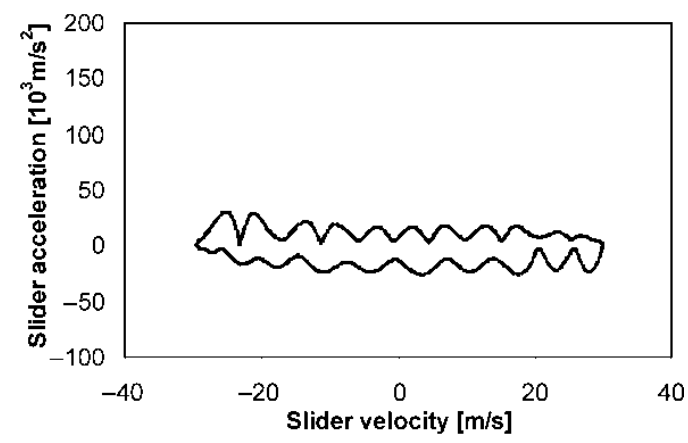

(d)

Fig. 13 Poincaré maps for different friction coefficients: (a) $c_{\mathrm{f}}=0.01$; (b) $c_{\mathrm{f}}=0.03$; (c) $c_{\mathrm{f}}=0.05$; (d) $c_{\mathrm{f}}=0.10$

The global results presented in this paper show that the non-periodic response in the mechanical systems can make the design and control processes more complex tasks. The dynamic behaviour of the multi-body systems with clearance joints is very sensitive to even small changes of parameters, namely the clearance size and the friction coefficient. Indeed, the system response can be periodic or chaotic with a very small variation of the initial parameters.

Most of the mechanical systems present some inherent non-linearity even when modelled with ideal joints. The non-linearity is more evident when the system includes clearance joints together with the friction effect. With the knowledge of non-linearities in multi-body systems, chaotic behaviour may be eliminated with suitable design and/or parameter changes of a mechanical system. In these cases, nonlinear control may be necessary to achieve the desired behaviour that may arise in the system. For the purpose of eliminating any non-linear behaviour that may arise in the mechanical systems, a non-linear controller must be employed to ensure dynamical stability.

\section{ACKNOWLEDGEMENTS}

The research work presented in this paper was supported by Fundação para a Ciência e a Tecnologia and partially financed by Fundo Comunitário Europeu
FEDER under project POCTI/2001/EME/38281, entitled 'Dynamic of Mechanical Systems with Joint Clearances and Imperfections'.

\section{REFERENCES}

1 Goodman, T. P. Dynamic effects of backlash. Mach. Des., 1963, 35, 150-157.

2 Dubowsky, S. and Freudenstein, F. Dynamic analysis of mechanical systems with clearances, part 1: formulation of dynamic model. J. Eng. Ind., 1971, 93(1), 305-309.

3 Dubowsky, S. and Freudenstein, F. Dynamic analysis of mechanical systems with clearances, part 2: dynamic response. J. Eng. Ind., 1971, 93(1), 310-316.

4 Schwab, A. L., Meijaard, J. P., and Meijers, P. A comparison of revolute joint clearance model in the dynamic analysis of rigid and elastic mechanical systems. Mech. Mach. Theory, 2002, 37(9), 895-913.

5 Flores, P., Ambrósio, J., and Claro, J. P. Dynamic analysis for planar multibody mechanical systems with lubricated joints. Multibody Syst. Dyn., 2004, 12, 47-74.

6 Lankarani, H. M. and Nikravesh, P. E. A contact force model with hysteresis damping for impact analysis of multibody systems. J. Mech. Des., 1990, 112, 369-376.

7 Ambrósio, J. A. C. Impact of rigid and flexible multibody systems: deformation description and contact models. In Virtual nonlinear multibody systems (Eds W. Schiehlen and M. Valásek), 2002, vol. II, pp. 15-33 (NATO, Advanced Study Institute, New York).

8 Flores, P. Dynamic analysis of mechanical systems with imperfect kinematic joints. PhD Dissertation, University of Minho, Guimarães, Portugal, 2005. 
9 Farahanchi, F. and Shaw, S. W. Chaotic and periodic dynamics of a slider crank mechanism with slider clearance. J. Sound Vib., 1994, 177(3), 307-324.

10 Rhee, J. and Akay, A. Dynamic response of a revolute joint with clearance. Mech. Mach. Theory, 1996, 31(1), 121-124.

11 Haines, R. S. An experimental investigation into the dynamic behaviour of revolute joints with varying degrees of clearance. Mech. Mach. Theory, 1985, 20(3), 221-231.

12 Soong, K. and Thompson, B. S. A theoretical and experimental investigation of the dynamic response of a slider-crank mechanism with radial clearance in the gudgeon-pin joint. J. Mech. Des., 1990, 112, 183-189.

13 Ravn, $P$. A continuous analysis method for planar multibody systems with joint clearance. Multibody Syst. Dyn., 1998, 2, 1-24.

14 Dubowsky, S. and Gardner, T. N. Design and analysis of multilink flexible mechanism with multiple clearance connections. J. Eng. Ind., 1977, 99(1), 88-96.

15 Dubowsky, S. and Moening, M. F. An experimental and analytical study of impact forces in elastic mechanical systems with clearances. Mech. Mach. Theory, 1978, 13, 451-465.

16 Dubowsky, S. On predicting the dynamic effects of clearances in one-dimensional closed loop systems. J. Eng. Ind., 1974, 96(1), 324-329.

17 Earles, S. W. E. and Wu, C. L. S. Motion analysis of a rigid link mechanism with clearance at a bearing using Lagrangian mechanics and digital computation. Mechanisms, 1973, 83-89.

18 Earles, S. W. E. and Wu, C. L. S. Predicting the occurrence of contact loss and impact at the bearing from a zero-clearance analysis. In Proceedings of IFToMM Fourth World Congress on the Theory of Machines and Mechanisms, Newcastle Upon Tyne, England, 1975, pp. 1013-1018.

19 Mahrus, D. Experimental investigation in to journal bearing performance. Rev. Bras. Tecnol., 1974, 5(3-4), $139-152$.

20 Wilson, R. and Fawcett, J. N. Dynamics of slider-crank mechanism with clearance in the sliding bearing. Mech. Mach. Theory, 1974, 9, 61-80.

21 Grant, S. J. and Fawcett, J. N. Effects of clearance at the Coupler-Rocker bearing of a 4-bar linkage. Mech. Mach. Theory, 1979, 14, 99-110.

22 Haines, R. S. Survey: 2-dimensional motion and impact at revolute joints. Mech. Mach. Theory, 1980, 15, $361-370$

23 Townsend, M. A. and Mansour, W. M. A pendulating model for mechanisms with clearances in the revolutes. J. Eng. Ind., 1975, 97(2), 354-358.

24 Miedema, B. and Mansour,W. M. Mechanical joints with clearance: a three mode model. J. Eng. Ind., 1976, 98(4), $1319-1323$.
25 Haines, R. S. A theory of contact loss at resolute joints with clearance. Proc. Instn Mech. Engrs, Part C: J. Mechanical Engineering Science, 1980, 22(3), 129-136.

26 Bengisu, M. T., Hidayetoglu, T., and Akay, A. A theoretical and experimental investigation of contact loss in the clearances of a four-bar mechanism. J. Mech. Transm. Autom. Des., 1986, 108, 237-244.

27 Feng, B., Morita, N., and Torii, T. A new optimization method for dynamic design of planar linkage with clearances at joints-optimizing the mass distribution of links to reduce the change of joint forces. J. Mech. Des., 2002, 124, 68-73.

28 Innocenti, C. Kinematic clearance sensitivity analysis of spatial structures with revolute joints. J. Mech. Des., 2002, 124, 52-57.

29 Orden, J. C. G. Analysis of joint clearances in multibody systems. Multibody Syst. Dyn., 2005, 13(4), 401-420.

30 Winfrey, R. C., Anderson, R.V., and Gnilka, C. W. Analysis of elastic machinery with clearances. J. Eng. Ind., 1973, 95, 695-703.

31 Dubowsky, S., Gu, P.Y., and Deck, J. F. The dynamic analysis of flexibility in mobile robotic manipulator systems. In Proceedings of VIII World Congress on the Theory of Machines and Mechanisms, Prague, Czechoslovakia, 26-31 August 1991, p. 12.

32 Bauchau, 0. A. and Rodriguez, J. Modelling of joints with clearance in flexible multibody systems. Int. J. Solids Struct., 2002, 39, 41-63.

33 Schwab, A. L. Dynamics of flexible multibody systems, small vibrations superimposed on a general rigid body motion. PhD Dissertation, Delft University of Technology, The Netherlands, 2002.

34 Kakizaki, T., Deck, J. F., and Dubowsky, S. Modeling the spatial dynamics of robotic manipulators with flexible links and joint clearances. J. Mech. Des., 1993, 115, 839-847.

35 Goldsmith, W. Impact, the theory and physical behaviour of colliding solids, 1960 (Edward Arnold Ltd, London, England).

36 Nikravesh, P. E. Computer-aided analysis of mechanical systems, 1960 (Prentice Hall, Englewood Cliffs, New Jersey).

37 Baumgarte, J. Stabilization of constraints and integrals of motion in dynamical systems. Comput. Methods Appl. Mech. Eng., 1972, 1, 1-16.

38 Shampine, L. and Gordon, M. Computer solution of ordinary differential equations: the initial value problem, 1972 (Freeman, San Francisco, California).

39 Baker, G. J. and Gollub, J. P. Chaotic dynamics - an introduction, 1990 (Cambridge University Press, Cambridge).

40 Tomsen, J. J. Vibrations and stability-order and chaos, 1990 (McGraw-Hill, New York).

41 Wiggins, S. Introduction to applied nonlinear dynamical systems and chaos, 1990 (Springer Verlag, New York). 